\title{
Spotlight on lenvatinib in the treatment of thyroid cancer: patient selection and perspectives
}

\author{
Ricardo Costa' \\ Benedito A Carneiro',2 \\ Sunandana Chandra ${ }^{1,2}$ \\ Sachin G Pai' \\ Young Kwang Chae ${ }^{1,2}$ \\ Jason B Kaplan ${ }^{1,2}$ \\ Hannah B Garrett' \\ Mark Agulnik ${ }^{1,2}$ \\ Peter A Kopp ${ }^{3}$ \\ Francis J Giles ${ }^{1,2}$ \\ 'Northwestern Medicine \\ Developmental Therapeutics Institute, \\ Robert H Lurie Comprehensive \\ Cancer Center of Northwestern \\ University, ${ }^{2}$ Division of Hematology \\ and Oncology, Feinberg School of \\ Medicine, ${ }^{3}$ Division of Endocrinology, \\ Metabolism, and Molecular Medicine, \\ Feinberg School of Medicine, \\ Northwestern University, Chicago, \\ IL, USA
}

Correspondence: Ricardo Costa Northwestern Medicine Developmental Therapeutics Institute, Robert H Lurie Comprehensive Cancer Center of Northwestern University, 233 East Superior Street, Olson Pavilion, Chicago, IL 606I I, USA

Tel + I 3124721234

$\mathrm{Fax}+\mathrm{I} 3124720564$

Email ricardo.costa@northwestern.edu
Abstract: Thyroid cancer is the most common endocrine malignancy, with over 60,000 cases reported per year in the US alone. The incidence of thyroid cancer has increased in the last several years. Patients with metastatic differentiated thyroid cancer (DTC) generally have a good prognosis. Metastatic DTC can often be treated in a targeted manner with radioactive iodine, but the ability to accumulate iodine is lost with decreasing differentiation. Until recently, chemotherapy was the only treatment in patients with advanced thyroid cancer, which is no longer amenable to therapy with radioactive iodine. The modest efficacy and significant toxicity of chemotherapy necessitated the need for urgent advances in the medical field. New insights in thyroid cancer biology propelled the development of targeted therapies for this disease, including the tyrosine kinase inhibitor sorafenib as salvage treatment for DTC. In 2015, the US Food and Drug Administration approved a second tyrosine kinase inhibitor, lenvatinib, for the treatment of radioiodine-refractory thyroid cancer. Although associated with a significant progression-free survival improvement as compared to placebo in a large Phase III study (median progression-free survival 18.2 vs 3.6 months; hazard ratio 0.21 ; $99 \%$ confidence interval $0.14-0.31 ; P<0.001$ ), the benefit of lenvatinib needs to be proved in the context of associated moderate to severe toxicities that require frequent dose reduction and delays. This article reviews the evidence supporting the use of lenvatinib as salvage therapy for radioactive iodine-refractory thyroid cancer, with a focus on the toxicity profile of this new therapy.

Keywords: lenvatinib, thyroid cancer, targeted therapy, differentiated thyroid cancer, tyrosine kinse inhibitor

\section{Introduction}

According to the Surveillance Epidemiology and End Results (SEER) database, the incidence of thyroid cancer, the most prevalent endocrine malignancy, increased in the US between 1975 and 2012, with estimated 62,450 new cases and 1950 deaths in $2015 .^{1}$ A rising incidence has also been documented in many other countries. ${ }^{2}$ The most common types of thyroid cancer arise from follicular cells, which include papillary thyroid carcinoma (PTC) and follicular thyroid carcinoma (FTC). These subtypes account for $90 \%-95 \%$ of all cases and are collectively referred to as differentiated thyroid cancers (DTCs). ${ }^{3}$ In general, these are indolent tumors associated with a favorable prognosis as reflected by long-term survival rates of $\sim 90 \%$ with standard treatments of surgery and radioactive iodine therapy. ${ }^{4}$ Nevertheless, patients who develop recurrent or metastatic radioactive iodine refractory disease have 10 -year survival rates of only $15 \%-20 \%{ }^{5}$

Before the advent of targeted therapies, chemotherapy was the only option for the treatment of patients with advanced thyroid cancer of follicular origin refractory to radioactive iodine. ${ }^{6}$ Doxorubicin (with its known cardiac and hematologic toxicities) alone or in combination with other compounds formed the backbone of palliative 
therapy for such patients. ${ }^{7,8}$ The oncogene pathway-driven approach to the understanding of the pathophysiology of thyroid cancer led to the development of clinical trials that assessed the antitumor activity of tyrosine kinase inhibitors (TKIs). These studies provided evidence of clinically meaningful antitumor activity that is not seen with chemotherapy and ultimately supported the US Food and Drug Administration's (FDA) approval of vandetanib and cabozantinib for the treatment of medullary thyroid cancer (MTC) and, more recently, sorafenib and lenvatinib for progressive radioiodine-refractory PTC and FTC. Notwithstanding their progression-free survival (PFS) benefit, TKIs are associated with significant and unique toxicity profiles. Furthermore, the decision of when to start treatment with a TKI can be challenging when encountering asymptomatic patients with slowly progressive radioiodine-refractory thyroid carcinomas, a relatively common finding in this disease. This article aims to critically review the data on antitumor activity, toxicity, and potential patient selection tools for the newly approved multikinase inhibitor lenvatinib. ${ }^{9}$

\section{Multikinase inhibitors approved for the treatment of follicular origin thyroid carcinomas}

A better understanding of the molecular biology of malignancies and the advent of targeted therapies represented an unprecedented development in the therapy of several solid tumors in recent years, including non-small-cell lung cancer, breast cancer, melanoma, and gastrointestinal stromal tumors. ${ }^{10-13}$ A comprehensive genetic analysis of 496 samples of PTC as part of the Cancer Genome Atlas (TCGA) project showed that driver genomic alterations were found in $\sim 97 \%$ of cases. ${ }^{14}$ The vascular endothelial growth factor receptor (VEGFR) was one of the first signaling pathways to be associated with the aggressiveness of thyroid cancer. ${ }^{15-17}$ Despite its key role in the pathophysiology of thyroid malignancies, other signaling pathways drive the thyroid cancer cell behavior. Fibroblast growth factor receptor (FGFR), plateletderived growth factor receptor (PDGFR), v-ras oncogene homologue (RAS), B-raf proto-oncogene, serine/threonine kinase (BRAF), and RET/PTC rearrangement receptor, among others, have been recognized as important signaling pathways that are implicated in the pathophysiology of thyroid tumors. ${ }^{18-24}$ Until recently, sorafenib was the only kinase inhibitor approved by the US FDA for the treatment of metastatic iodine-refractory DTC. Sorafenib is an oral TKI that abrogates signaling from numerous molecules including BRAF, RET/PTC, VEGFR1-3, PDGFR, and c-KIT. ${ }^{25,26}$
The antineoplastic activity of sorafenib for the treatment of thyroid cancer was demonstrated in many trials including the DECISION trial, which was a Phase III placebo-controlled randomized study of 417 patients with progressive radioactive iodine-refractory, locally advanced, or metastatic thyroid cancer assigned to sorafenib $400 \mathrm{mg}$ twice daily or placebo. ${ }^{27,28}$ The histological subtypes, confirmed by a central review, primarily consisted of PTC and FTC. The primary end point of the study was met with a hazard ratio (HR) for PFS of 0.59 (95\% confidence interval [CI] 0.45-0.76; $P<0.0001$ ). The overall survival (OS) difference did not meet statistical significance, likely due to a crossover effect (HR 0.80; 95\% CI 0.54-1.19; $P=0.14$ ). These benefits were counterbalanced by frequent treatment-related adverse events (AEs) including hand-foot syndrome (HFS), diarrhea, alopecia, and rash, which were documented in $\sim 70 \%$ of the participants. Serious AEs occurred in $37 \%$ of patients treated with sorafenib compared to $26 \%$ of those treated with placebo. The most common treatment-related grade 3 AEs in the sorafenib arm included HFS (20\%), diarrhea (5\%), skin rash or desquamation (4.8\%), fatigue (5\%), and weight loss (5.8\%). Dose modifications because of adverse effects were required in $\sim 60 \%$ of the patients, and the treatment was suspended in $18 \%$ of the patients. HFS was the most frequent AE leading to dose modifications. These results are consistent with the toxicity profile of sorafenib observed in other malignancies.

Most recently, a meta-analysis of 24 Phase II and III trials estimated the risk of all-grade HFS at $39 \%$ for patients treated with sorafenib. ${ }^{29}$ Prophylactic treatment with urea-based cream can decrease the incidence of or delay the development of HFS in patients receiving sorafenib. ${ }^{30}$ Another metaanalysis showed that all-grade diarrhea occurred in $37 \%$ of patients treated with sorafenib for different types of tumors and can negatively impact quality of life. ${ }^{31}$

In addition to sorafenib, several other kinase inhibitors have been studied for the treatment of advanced follicular origin radioiodine-refractory thyroid cancer (Table 1).

\section{Lenvatinib: overview}

Lenvatinib is a TKI that targets VEGFR1-3, FGFR1-4, PDGFR- $\alpha$, RET, and c-KIT. ${ }^{32-35}$ Preclinical evidence indicates that lenvatinib inhibits tumor growth, tumor-induced angiogenesis, cell migration, and invasion in various human thyroid cancer xenograft and in vitro models. ${ }^{36,37}$ Compared to the other multikinase inhibitors tested in patients with differentiated thyroid tumors, lenvatinib is particularly active against the FGFR family of tyrosine kinase receptors 


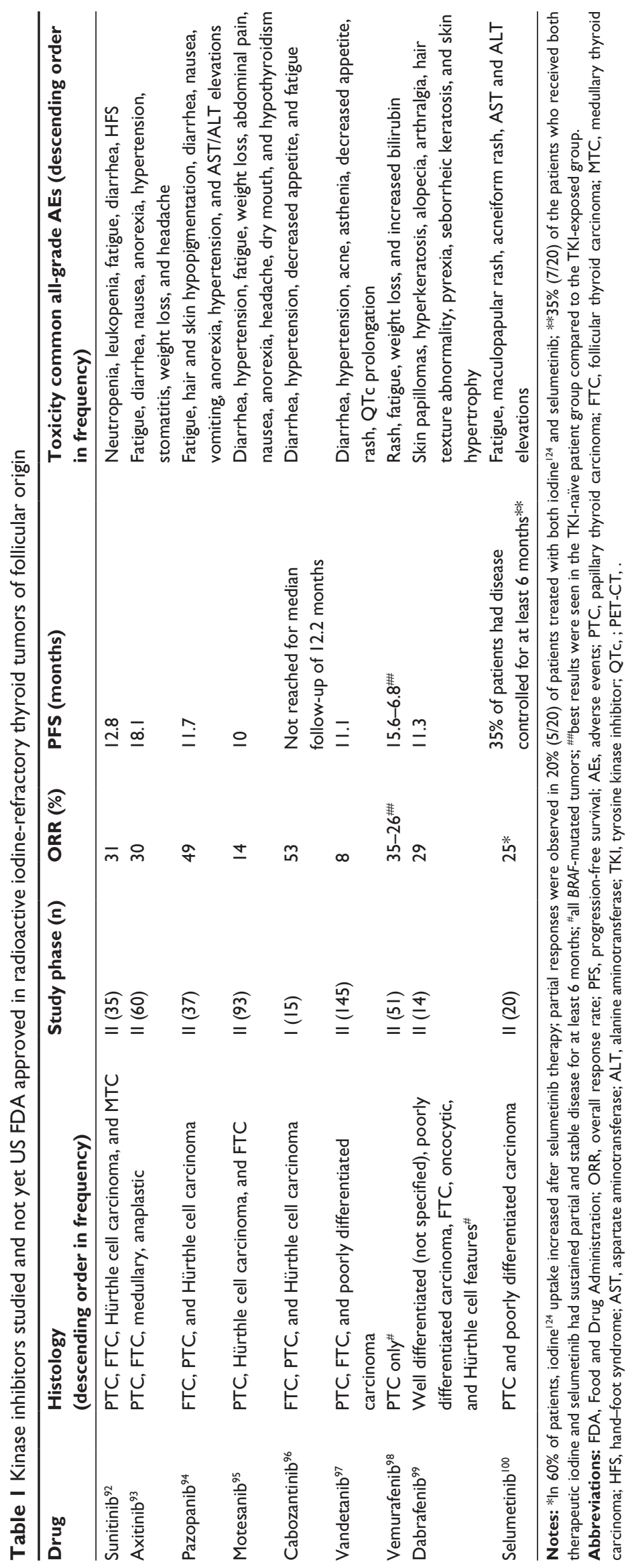


(Table 2). FGFRs contain three immunoglobulin-like extracellular domains, a transmembrane region, and an intracellular domain, which is composed of a split tyrosine kinase and its carboxyterminus. ${ }^{38}$ FGFRs are overexpressed in a variety of solid tumors, including thyroid cancer. ${ }^{39,40}$ Preclinical models and immunohistochemistry analyses of tumor tissues showed that FGFR1 is overexpressed in $50 \%-75 \%$ of cells in both follicular and papillary tumors. FGFR2 seems to be expressed in normal thyroid tissues but not in tumors. ${ }^{19}$ FGFR3 is overexpressed in 25\%-50\% of well-differentiated follicular origin thyroid cancers. Cells and xenograft models were treated with the TKI PD173074, which is known to selectively inhibit FGFR phosphorylation and tyrosine kinase activity in solid tumors. ${ }^{41}$ The abrogation of FGFR1 and 3 signaling with PD173074 was associated with in vitro and in vivo growth inhibition of differentiated thyroid tumor cell lines. TPC-1 cells sustained up to $90 \%$ inhibition of cell proliferation, and xenograft models treated with PD173074 achieved a tumor size reduction of $\sim 55 \%{ }^{19}$ These observations indicate the importance of the FGFR pathway in thyroid cancer pathogenesis and progression. Next-generation sequencing analysis of the genomes of 492 samples of PTC identified this disease as having a low mutational burden compared with other carcinomas. ${ }^{14}$ FGFR3 amplification was noted in only one case, and FGFR2 gene fusions were observed in only two cases. Evidence supports that FGFR overexpression is regulated through epigenetic modulation. ${ }^{42}$

\section{Clinical efficacy and toxicity of lenvatinib}

In a dose-escalation 3+3 Phase I trial, the maximum tolerated dose (MTD) of lenvatinib was investigated among a wide range of doses ( $0.5-20 \mathrm{mg}$ twice daily) in solid tumors, including colon, sarcoma, and non-small-cell lung cancers. ${ }^{43}$ Dose-limiting toxicities of grade 3 elevations of aspartate and alanine aminotransferases and thrombocytopenia were observed at the $20 \mathrm{mg}$ twice-daily dose level. MTD was $13 \mathrm{mg}$ twice daily on a 2-week-on/1-week-off schedule for this trial. Of note, five patients experienced six serious AEs related or possibly related to the study drug. ${ }^{43}$ A larger Phase I trial investigated the safety and efficacy of lenvatinib among 82 patients with advanced refractory solid tumors, which included renal, colorectal, and pancreatic tumors. Lenvatinib was well tolerated at $25 \mathrm{mg}$ daily. Grade 3 hypertension and proteinuria occurred in nine patients $(11 \%)$ and six patients (7\%), respectively. A positive correlation between the dose of lenvatinib and hypertension and proteinuria was observed. In the expanded MTD cohort of $25 \mathrm{mg}(\mathrm{n}=24)$, grade 3 hypertension occurred in three $(13 \%)$ patients and grade 3 proteinuria in two $(8 \%)$ patients. ${ }^{44}$

Based on these Phase I trial results, a subsequent Phase II trial was conducted among 58 patients with differentiated radioiodine-refractory thyroid cancer who were treated with lenvatinib at $24 \mathrm{mg}$ daily. Prior anti-VEGFR therapy and chemotherapy were reported in $29 \%$ and $14 \%$ of the patients, respectively. There was a response rate of $50 \%$ and a median PFS of 12.7 months. AEs led to dose interruptions, reductions, or study drug withdrawal in $74 \%, 66 \%$, and $26 \%$ of the patients, respectively. Grade 3 or higher AEs occurred in $72 \%$ of the patients. The most common grade 3 side effects were weight loss (12\%), hypertension $(10 \%)$, proteinuria $(10 \%)$, diarrhea $(10 \%)$, fatigue (9\%), dehydration (9\%), and arthralgia (5\%). Of note, two patients died after serious AEs (one arterial hemorrhage and one cardiac arrest). ${ }^{45}$

In another cohort of patients, lenvatinib was tested at the set dose of $24 \mathrm{mg}$ daily in 59 patients with advanced

Table 2 Kinase inhibitors studied in radioiodine-refractory PTC and FTC and their targets

\begin{tabular}{|c|c|c|c|c|c|c|c|c|c|c|c|}
\hline \multirow[t]{2}{*}{ TKI } & \multicolumn{11}{|l|}{ Targets } \\
\hline & VEGFR & RET & C-KIT & PDGFR & FGFR & BRAF & EGFR & MET & FLT3 & MEKI & MEK2 \\
\hline Lenvatinib $32,33,36$ & $\checkmark$ & $\checkmark$ & $\checkmark$ & $\checkmark$ & $\checkmark$ & & & & & & \\
\hline Sorafenib ${ }^{25,26}$ & $\checkmark$ & $\checkmark$ & & $\checkmark$ & & $\checkmark$ & & & $\checkmark$ & & \\
\hline Sunitinib ${ }^{101}$ & $\checkmark$ & & $\checkmark$ & $\checkmark$ & & & & & & & \\
\hline Axitinib $^{102}$ & $\checkmark$ & & $\checkmark$ & $\checkmark$ & & & & & & & \\
\hline Pazopanib 103,104 & $\checkmark$ & & $\checkmark$ & $\checkmark$ & & & & & & & \\
\hline Motesanib $^{105}$ & $\checkmark$ & $\checkmark$ & $\checkmark$ & $\checkmark$ & & & & & & & \\
\hline Cabozantinib 106,107 & $\checkmark$ & $\checkmark$ & $\checkmark$ & & & & & $\checkmark$ & $\checkmark$ & & \\
\hline$V^{\text {Vandetinib }}{ }^{108,109}$ & $\checkmark$ & $\checkmark$ & & & & & $\checkmark$ & & & & \\
\hline Dabrafenib 110 & & & & & & $\checkmark$ & & & & & \\
\hline Selumetinib"II & & & & & & & & & & $\checkmark$ & $\checkmark$ \\
\hline Vemurafenib $1 / 2$ & & & & & & $\checkmark$ & & & & & \\
\hline
\end{tabular}

Abbreviations: PTC, papillary thyroid carcinoma; FTC, follicular thyroid carcinoma; VEGFR, vascular endothelial growth factor receptor; PDGFR, platelet-derived growth factor receptor; FGFR, fibroblast growth factor receptor; EGFR, epidermal growth factor receptor; FLT3, FMS-like tyrosine kinase 3. 
MTC, 44\% of whom received prior anti-VEGFR therapy and $15 \%$ received prior chemotherapy. ${ }^{46}$ Overall response rate (ORR) was 36\%, and median PFS was 9 months. Dose reduction or interruption was necessary in up to $59 \%$ of the patients, and $24 \%$ of the patients discontinued treatment due to AEs. The most common grade 3 side effects included diarrhea (14\%), hypertension (7\%), decreased appetite (7\%), fatigue $(5 \%)$, dysphagia (5\%), and increased levels of alanine aminotransferase (5\%). Overall, grade 3 AEs occurred in 36 patients $(61 \%)$. There were three deaths due to AEs including respiratory arrest (not otherwise specified), respiratory failure, and paraneoplastic syndrome (not specified); only the respiratory failure-related death was deemed to be treatment related by the treating physicians. ${ }^{46}$

In the seminal Phase III study SELECT, a total of 392 patients with radioactive iodine-resistant progressive thyroid carcinomas of follicular origin (defined as progression within the previous 13 months according to Response Evaluation Criteria In Solid Tumors, the presence of one measurable lesion without radioiodine uptake on any ${ }^{131}$ iodine scan, or disease progression within 12 months of radioiodine therapy) were randomized to either lenvatinib $(n=261)$ or placebo $(\mathrm{n}=131) .{ }^{47}$ A significant improvement in the median PFS, the primary end point, was documented among patients treated with lenvatinib compared to placebo (18.3 vs 3.6 months; HR $0.21 ; 99 \%$ CI $0.14-0.31 ; P<0.001)$, with an unprecedented response rate of $64.8 \%$. Ninety-three patients in the trial received prior anti-VEGF TKIs (sorafenib 77\%, sunitinib $9 \%$, pazopanib 5\%, and other $9 \%$ ). Despite the previous use of targeted therapies, lenvatinib also significantly improved the PFS in this subgroup of patients (15.1 vs 3.6 months; HR 0.22 ; 95\% CI 0.12-0.41) and increased response rates (62.1 vs. $3.7 \%$ \%). ${ }^{48}$ Complete radiologic responses were seen in four patients, and prolonged stable disease (longer than 23 weeks) was noted in 39 patients treated with lenvatinib. While consistent with the results of Phase I and II studies, the toxicity profile in this study was significant.

As noted with the use of other multikinase inhibitors, lenvatinib toxicity resulted in a significant rate of dose adjustments and clinically relevant consequences. Grade 3 or higher toxicities were seen in $75 \%$ of the patients. Dose reductions, dose interruptions, and treatment discontinuation were needed in $67 \%, 82 \%$, and $14 \%$ of the patients, respectively. The most frequent grade 3 or higher treatment-related AEs were hypertension (42\%), fatigue (9\%), diarrhea (8\%), proteinuria $(10 \%)$, arterial and venous thromboembolic effects $(2.7 \%$ and $3.8 \%$, respectively), acute renal failure $(1.9 \%)$, and hepatic failure $(0.4 \%)$. Dose modifications due to severe diarrhea and decreased appetite were required in
$22 \%$ and $18 \%$ of the patients, respectively. In this larger trial, QTc prolongation was seen in $8 \%$ of the patients, of whom $1.5 \%$ experienced grade $\geq 3$ AEs, defined as a QTc $\geq 501$ ms. However, among a study of healthy individuals who took a single $32 \mathrm{mg}$ dose of lenvatinib, there was a lack of QTc prolongation within the 24-hour continuous electrocardiogram (EKG) monitoring period..$^{49}$ Thus far, there seems to be no evidence of significant direct cardiac toxicity associated with lenvatinib.

Of note, six deaths in the lenvatinib group were considered treatment related: three cases resulted from unspecified causes and three were associated with pulmonary embolism, hemorrhagic stroke, and health deterioration. However, four of these deaths were nonspecific in etiology, and it is thus difficult to accurately assess the contribution of lenvatinib toxicity to these fatal events.

As of yet, data on median OS are not available, and no significant OS benefit has been demonstrated with lenvatinib thus far (HR $0.73,95 \%$ CI $0.5-1.0 ; P=0.10$ ). Notably, 109 patients per protocol were allowed to cross over to the lenvatinib arm upon disease progression, which can confound differences in OS. As of November 2013, 130 patients received blinded treatment. Nonetheless, an updated survival data analysis of the SELECT trial at a later cut-off date (June 15, 2014) was presented at the 2015 European Cancer Congress. ${ }^{50}$ To adjust for the crossover and estimate the true OS treatment effect (the effect that would have been observed in the absence of switching), a rank-preserving structural failure time model was used. After 34 months of follow-up of the trial for the lenvatinib arm, the median OS was not reached for this group. For the placebo crossover arm, the reported medial OS was 19.1 months (95\% CI 14.3, not estimable). The rank-preserving structural failure time-adjusted HR showed a significant difference in OS between the treatment groups (HR 0.53; 95\% CI 0.34-0.82; nominal $P=0.0051$ ) as determined using the resampling method (bootstrapping).

Of note, hypertension was the most common grade 3 or higher toxicity, affecting $42 \%$ of the patients in the SELECT trial, while observed in only $7 \%-10 \%$ of the patients in the Phase II trials. ${ }^{45,46}$ All-grade hypertension occurred in up to $67 \%$ of the patients treated with lenvatinib (SELECT), and it led to dose reductions or interruptions in up to $20 \%$ of the patients. In the Phase II trials, hypertension was observed in $51 \%-76 \%$ of the patients treated with lenvatinib. Because of the risk of hypertension, careful cardiovascular evaluation of patients, including performing an echocardiogram and EKG profile, should be considered prior to initiation of therapy. An EKG should also be performed prior to initiation of therapy, considering the risk of QTc prolongation associated with multikinase inhibitors in general. ${ }^{51}$ 
Frequent blood pressure monitoring should be performed throughout treatment. According to the SELECT study protocol, dose interruptions of up to 28 days for patients with grade $\geq 2$ hypertension should be performed prior to dose reduction (20, 14, $10 \mathrm{mg} / \mathrm{d})$. Patients with confirmed systolic blood pressure $(\mathrm{BP}) \geq 140 \mathrm{mmHg}$ or diastolic $\mathrm{BP} \geq 90 \mathrm{mmHg}$ should be prescribed antihypertensive agents and monitored every 2 weeks. Patients with systolic BP $\geq 160 \mathrm{mmHg}$ or diastolic $\mathrm{BP} \geq 100 \mathrm{mmHg}$, despite optimal management, should have a dose reduction.

In the Phase III SELECT study, gastrointestinal perforation or fistula was observed in $2 \%$ of patients receiving lenvatinib compared to $0.8 \%$ in the placebo group. ${ }^{52}$ In addition, aerodigestive fistula formation had also been documented in a case report in which a patient receiving lenvatinib developed significant general decline and required percutaneous gastrostomy for nutritional support. ${ }^{53}$ Radiation therapy and large thoracic tumor burden are considered risk factors for aerodigestive fistula formation associated with lenvatinib and other anti-angiogenesis therapies. ${ }^{54,55}$ Hemorrhagic complications were also documented in the $35 \%$ of patients treated with lenvatinib in the Phase III trial compared to $18 \%$ in the placebo group. Epistaxis was the most common hemorrhagic event ( $11 \%$ grade 1 and $1 \%$ grade 2$).{ }^{52}$ The incidence of severe hemorrhage episodes (grade 3-5) was comparable between both the groups (2\% lenvatinib and 3\% placebo). One patient with brain metastases at baseline treated with lenvatinib died from intracranial hemorrhage. These results are in agreement with the risk of fatal bleeding documented with other tyrosine kinase inhibitors and highlight the importance of careful monitoring. ${ }^{56}$ The clinically meaningful benefit in PFS and tumor response rate in this disease setting led to the US FDA's approval of lenvatinib in February 2015 for the treatment of progressive thyroid cancer.

\section{Potential predictive biomarkers for lenvatinib efficacy}

Despite the overall response rate of $>60 \%$ across distinct histologic subtypes (ie, papillary, poorly differentiated, follicular, and Hürthle cell), up to $35 \%$ of the patients did not meet criteria for response under the therapy with lenvatinib. ${ }^{47}$ This is particularly important in patients who are symptomatic from their tumor burden and would benefit from tumor reduction. Also, as described earlier, lenvatinib has been associated with significant toxicity and treatment-related deaths. Hence, it is paramount to find predictive markers of response, which may facilitate selecting patients for therapy.
The first and best-characterized point mutation in thyroid cancer is a valine-to-glutamate substitution at residue 600 (V600E) of the BRAF (v-raf murine sarcoma viral oncogenes homolog B1) gene. ${ }^{14,57}$ This results in constitutive activation of the BRAF kinase that confers continuous activation of the mitogen-activated protein kinase (MAPK) signaling pathway with consequent uncontrolled cell growth. ${ }^{58} B R A F$ mutations may have prognostic value in differentiated thyroid tumors. A recent retrospective analysis of 500 patients with PTC showed tumor recurrence rates of $25 \%$ among those with a $B R A F \mathrm{~V} 600 \mathrm{E}$ mutation as compared to $9.6 \%$ in mutation-negative patients. ${ }^{59}$

Following $B R A F$ mutations in thyroid cancers (40\%), the most frequent driver mutations occur in the $R A S$ genes. There are three isoforms of $R A S$ : HRAS, KRAS, and NRAS. The most common $R A S$ mutations in thyroid cancer occur in the NRAS and HRAS genes and lead to constitutive activation of both the MAPK and phosphoinositide 3-kinase (PI3K)/ AKT pathways. ${ }^{60,61} R A S$ can be mutated in up to $20 \%$ of FTC and in 6\%-13\% of patients with PTC. ${ }^{14,60,61}$ Archival formalinfixed, paraffin-embedded tissues were obtained and analyzed for $B R A F$ and $R A S$ mutation hotspots in the SELECT trial. Interestingly, the benefit of lenvatinib was independent of the $B R A F$ and RAS mutational status of the tumor on a preplanned subgroup analysis in SELECT. ${ }^{47}$ This could be due to the wide range of tumor cell targets affected by lenvatinib or its ability to target unique molecules such as the FGFR. ${ }^{19}$

The angiogenesis of MTC, a highly vascular tumor, is mediated principally by VEGF, FGF, PDGF, and their respective receptors. ${ }^{62}$ Overexpression of VEGF and VEGFR2 has been observed in 50\%-95\% of MTC tumors and is associated with metastases. ${ }^{63-65}$ In the aforementioned Phase II trial of lenvatinib for the treatment of 59 patients with advanced MTC, in which circulating cytokine and angiogenic factors levels were collected from 51 patients after 8 days of therapy, high baseline levels of VEGF, soluble VEGFR3, and PDGF- $\beta$, and low baseline levels of soluble Tie-2 were associated with tumor reduction. Low baseline levels of angiopoietin-2 (Ang-2), hepatocyte growth factor, and interleukin- 8 were associated with tumor reduction and prolonged PFS. ${ }^{46}$ In the SELECT trial, which accrued patients with FTC and PTC, blood cytokine and angiogenic factor levels of $99 \%$ of patients were collected on cycle 1 , day 15 and day 1 of subsequent cycles. Ang-2 and sTie-2 levels were consistently decreased throughout the therapy with lenvatinib when compared with the placebo group. Decreased Ang-2 and sTie-2 levels along with increased VEGF levels correlated with tumor shrinkage; at the end of the treatment, the levels of these two molecules on C2D1 
increased in $78.9 \%$ and $81 \%$ of patients, respectively. ${ }^{66}$ The association between lenvatinib benefit or lack thereof and baseline angiogenic factors merits further investigation before it can affect patient treatment selection.

Finally, in light of lenvatinib-related toxicity, the possible interaction between age and therapy with lenvatinib was formally explored for OS end point in the SELECT trial. Improved OS was identified in patients older than 65 years (median 71 years) when treated with lenvatinib. ${ }^{67}$

\section{Discussion}

At this juncture, the antitumor activity of lenvatinib, including its improvement in PFS, among patients with advanced progressive thyroid carcinomas of follicular origin supports its clinical use. Recently published guidelines by the American Thyroid Association and the National Comprehensive Cancer Network recommend its possible use in patients with radioiodine-refractory progressive thyroid carcinomas and as first-line kinase inhibitor therapy. ${ }^{68,69}$

Thus far, no other multikinase inhibitors have displayed a comparable degree of efficacy in the treatment of advanced radioiodine-refractory thyroid cancer. Taking into account all of the caveats inherent to cross-trial comparisons, the US FDA approved the TKI sorafenib based on the results of the DECISION trial, which showed a PFS of 10.8 months in the sorafenib arm vs 5.8 months in the placebo arm (HR 0.59; $95 \%$ CI $0.45-0.76 ; P<0.0001)$ and an ORR of $12.2 \% .{ }^{27}$ By contrast, lenvatinib-treated patients derived a greater benefit from this TKI compared to placebo in the SELECT trial, as reflected by a PFS of 18.3 months vs 3.6 months (HR $0.21 ; 99 \%$ CI $0.14-0.31 ; P<0.001)$ and an ORR of $64.8 \% .{ }^{47}$ Both these trials included similar patient populations, but the DECISION trial excluded patients with previous TKI exposure. The SELECT trial included 93 patients who had received prior TKI therapy (sorafenib 77\%, sunitinib 9\%, pazopanib $5 \%$, other $9 \%$ ). The benefit of lenvatinib was not mitigated by prior multikinase treatment. ${ }^{48}$ One could hypothesize that the remarkable antitumor activity of lenvatinib is due to its tyrosine kinase inhibition of FGFR ${ }^{19}$ FGFR family activation leads to upregulation of the RAS/MAPK and PI3K/AKT signaling pathways. ${ }^{70}$ FGFR overexpression and phosphorylation observed in preclinical studies suggest that FGFR is an important signaling pathway in the thyroid tumors of follicular origin, as reviewed earlier. ${ }^{19}$

Despite its efficacy, the toxicity of lenvatinib merits cautious consideration. Up to $65 \%-71 \%$ of the patients treated with lenvatinib experienced grade 3 or higher toxicities, having warranted dose reductions and/or delays in the majority of the patients in previous Phase II and III clinical trials (Table 3). ${ }^{45-47}$ The pattern of disease progression in radioiodine-refractory thyroid tumor should also be considered at the time of therapy initiation (median PFS in placebo-treated groups range from 3.6-5.8 months) to the extent that asymptomatic patients may take months to meet criteria for disease progression. Hence, the potential for antitumor efficacy needs be carefully counterbalanced against its potential toxicities in asymptomatic patients prior to drug initiation. Lenvatinib should be initiated at the time of rapid radiological/clinical disease progression and in the setting of symptomatic tumor burden. Furthermore, patients need to be carefully selected for lenvatinib therapy, and physicians should be aware of the frequency of moderate to severe AEs, including hypertension, weight loss, decreased appetite, and proteinuria associated with this medication (Table 3 ).

Finally, careful consideration of locally directed therapies (eg, surgery, radiation therapy, thermal ablation) should be given prior to initiation and during the course of treatment with lenvatinib. ${ }^{68,69}$ This is of particular importance in the setting of mixed responses in distinct metastatic sites with imminent risk of complications related to local progression and invasion of metastasis (Figure 1).

Formal quality of life assessments were not reported for the SELECT trial, but sorafenib-treated patients in the DECISION trial had lower on-treatment scores for the Functional Assessment of Cancer Therapy: General, EuroQol-5D and EuroQol-5D visual analog scales, suggesting a detectable negative impact of sorafenib therapy on health-related quality of life. ${ }^{71}$ Nonetheless, in light of the toxicity profile, the corollary is that physicians need to be fully aware and aggressively treat eventual AEs to maintain quality of life.

The role of lenvatinib as salvage therapy for patients with previous exposure to TKIs, such as sorafenib, is a question of clinical relevance. A retrospective analysis of 17 patients with sorafenib-refractory thyroid cancer treated with salvage therapy, including sunitinib $(n=4)$, pazopanib $(n=3)$, cabozantinib $(n=4)$, lenvatinib $(n=3)$, and vemurafenib $(n=3)$, revealed an ORR of $41 \%$ and a median PFS of 11.4 months. ${ }^{72}$ This suggests that TKIs with different targets may still be effective for refractory disease. These results are consistent with a subgroup analysis of the SELECT trial in which patients who had been previously exposed to TKIs still derived benefit from lenvatinib. ${ }^{48}$ Patients with a history of previous exposure to TKIs had an ORR of $65.6 \%$, whereas TKI-naïve patients had an ORR of $62.1 \%$.

The question of whether lenvatinib or sorafenib should be the first option for first-line treatment of patients with 
iodine-refractory DTC still remains. The lack of head-tohead comparison of these molecules generates the need for constant individualized decision making, taking into account the estimated efficacy and toxicity for each multikinase inhibitor.

There is no biomarker that facilitates the selection of or the prediction of response to lenvatinib. Stratified analysis of pre-therapy $B R A F$ and $R A S$ mutational status failed to show a predictive benefit of these tests in Phase III trials of sorafenib and lenvatinib. 27,47

Simultaneous targeting of multiple molecular pathways is a potential strategy to improve the antitumor activity of lenvatinib and to potentially increase the efficacy of and delay resistance to lenvatinib therapy in radioiodine-refractory thyroid cancer. The combined analysis of genomic variants, gene expression, and methylation patterns of 496 samples of PTC was able to cluster this tumor into two main molecular signature groups: the $B R A F$-mutant and the $R A S$-mutant tumors with differential downstream pathway activation of MAPK and PI3K/AKT, respectively. ${ }^{14}$ One could hypothesize that lenvatinib's efficacy could be further increased by treatment combinations driven by pathway-enriched patient selection according to differential tumor signatures. The development of the combination treatment with the MEK inhibitor trametinib and the $B R A F$ inhibitor dabrafenib in recurrent thyroid cancer is ongoing (NCT01723202) ${ }^{73}$ However, to our knowledge, the multiple pathway blockade approach with lenvatinib is nonexistent at this time.

Also, hepatocyte growth factor (HGF) and Met are overexpressed in the majority of PTCs $(>90 \%)$, but rarely in FTCs. ${ }^{74} \mathrm{HGF}$ overexpression correlates with invasiveness and a more clinically aggressive behavior of PTCs. ${ }^{75-77}$ The Met receptor is a key component of HGF pathobiology in PTC. ${ }^{78}$ Among PTC cell lines, the Met inhibitor PHA665752 inhibited tumor cell growth and induced apoptosis. ${ }^{79}$ Interestingly, preclinical data indicate that one of the mechanisms of intrinsic lenvatinib therapy resistance is mediated by HGF pathway activation in various solid tumors such as melanoma and pancreatic carcinoma. ${ }^{80}$ This can be circumvented by the combination of lenvatinib with the Met inhibitor golvatinib, which showed significant reduction of lenvatinib-resistant cell proliferation in vitro. ${ }^{80} \mathrm{~A}$ Phase I dose-escalation trial of the combination of lenvatinib and golvatinib in patients with melanoma and glioblastoma is currently under way (NCT01433991). ${ }^{81}$ Further studies are necessary to establish the role of Met inhibition in thyroid cancer.

Interestingly, the degree of toxicity from anti-endothelial growth factor receptor and anti-VEGF drugs has been 


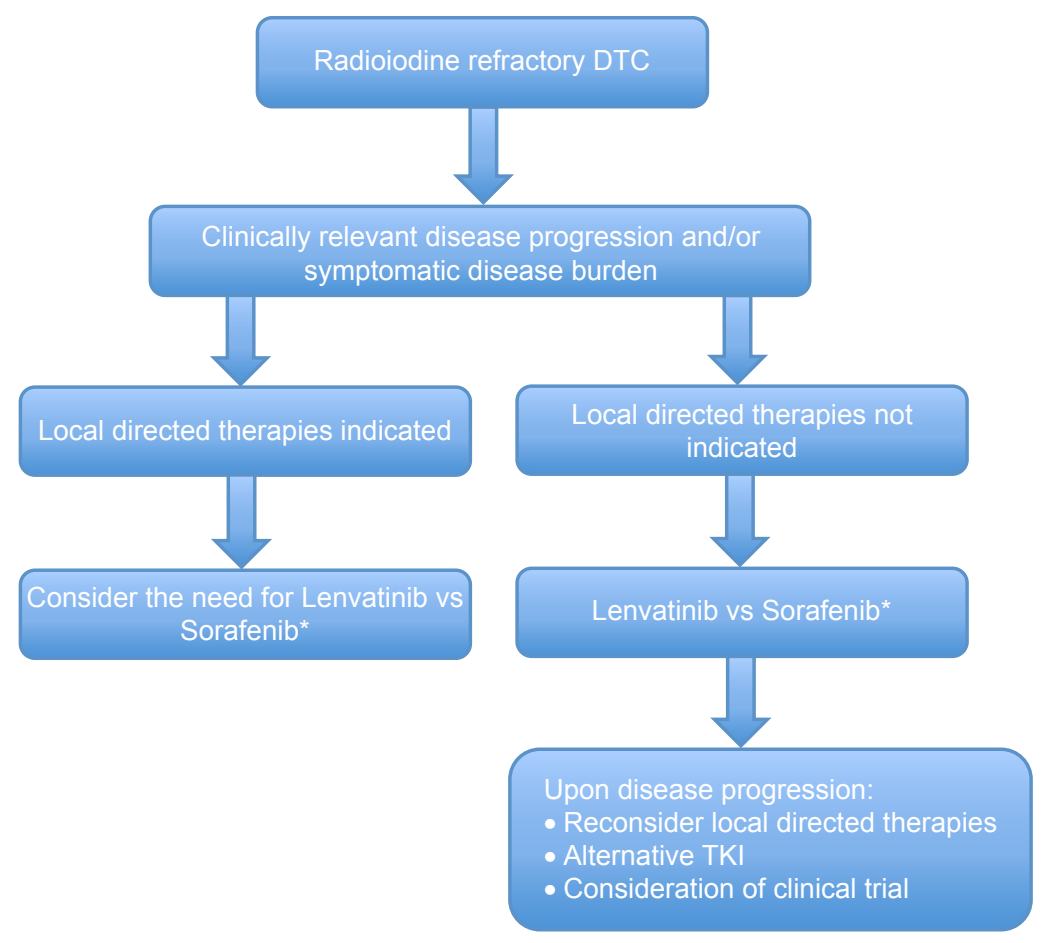

Figure I Proposed algorithm for initiation of lenvatinib therapy in patients with DTC. Note: *Individualized decision making is necessary.

Abbreviations: DTC, Differentiated thyroid cancer; TKI, Tyrosine kinase inhibitor.

positively correlated with their efficacy among patients with solid tumors including colorectal, lung, renal cell carcinoma, and head and neck. ${ }^{82,83}$ The degree of toxicity, namely hypertension and proteinuria, could serve as a surrogate biomarker of lenvatinib activity. It has been suggested that AEs such as worst grade proteinuria correlated with lenvatinib antitumor activity in a small Phase I dose-escalation clinical trial. ${ }^{84}$ To our knowledge, no stratified analysis of the efficacy of lenvatinib by toxicity has been reported.

In addition, dose reduction of TKIs positively correlated with clinical benefit in non-small-cell lung cancer and chronic myeloid leukemia. ${ }^{85-87}$ In clinical practice, it is tempting to adopt a dose reduction strategy to avoid lenvatinib-associated toxicities while offering patients effective treatment. Data are not yet available on the efficacy of dose-reduced treatment of advanced thyroid cancer with lenvatinib. The results of the expanded cohort program with lenvatinib for the treatment of radioiodine-refractory DTC with three different doses, 24,20 , and $14 \mathrm{mg}$ daily will shed light into this important question (NCT02211222). ${ }^{88}$

In conclusion, lenvatinib is a novel multikinase inhibitor that demonstrates significant responses and PFS benefit in the treatment of radioiodine-refractory DTC. Consistent with the current 2015 American Thyroid Association and the National Comprehensive Cancer Network guidelines, lenvatinib should be considered as the first-line therapy in the setting of clinically relevant disease progression and/or symptomatic disease burden in the setting of radioiodinerefractory disease, with the warning that physicians should be fully cognizant of its side effect profile, including potentially fatal toxicities (Figure 1). ${ }^{68,69}$ Aggressive monitoring and guideline-based management of toxicities similar to those utilized with other TKIs should be enforced to prevent decline in quality of life. ${ }^{89-91}$ Future studies should endeavor to establish biomarkers predictive of toxicity and efficacy of lenvatinib treatment in radioiodine-refractory thyroid tumors.

\section{Disclosure}

The authors report no conflicts of interest in this work.

\section{References}

1. Institute NC. SEER Cancer Statistics Factsheets: Thyroid Cancer. National Cancer Institute. Bethesda, MD. Available from: http://seer. cancer.gov/statfacts/html/thyro.html. Accessed October 10, 2015.

2. Pellegriti G, Frasca F, Regalbuto C, Squatrito S, Vigneri R. Worldwide increasing incidence of thyroid cancer: update on epidemiology and risk factors. J Cancer Epidemiol. 2013;2013:965212.

3. American Thyroid Association Guidelines Taskforce on Thyroid Nodules and Differentiated Thyroid Cancer, Cooper DS, Doherty GM, et al. Revised American Thyroid Association management guidelines for patients with thyroid nodules and differentiated thyroid cancer. Thyroid. 2009;19(11):1167-1214. 
4. Brenner $H$. Long-term survival rates of cancer patients achieved by the end of the 20th century: a period analysis. Lancet. 2002;360(9340): 1131-1135.

5. Durante C, Haddy N, Baudin E, et al. Long-term outcome of 444 patients with distant metastases from papillary and follicular thyroid carcinoma: benefits and limits of radioiodine therapy. J Clin Endocrinol Metab. 2006;91(8):2892-2899.

6. Carter SK, Blum RH. New chemotherapeutic agents - bleomycin and adriamycin. CA Cancer J Clin. 1974;24(6):322-331.

7. Gottlieb JA, Hill CS Jr. Chemotherapy of thyroid cancer with adriamycin. Experience with 30 patients. N Engl J Med. 1974;290(4):193-197.

8. Shimaoka K, Schoenfeld DA, DeWys WD, Creech RH, DeConti R. A randomized trial of doxorubicin versus doxorubicin plus cisplatin in patients with advanced thyroid carcinoma. Cancer. 1985;56(9): 2155-2160.

9. Nair A, Lemery SJ, Yang J, et al. FDA approval summary: lenvatinib for progressive, radio-iodine-refractory differentiated thyroid cancer. Clin Cancer Res. 2015;21(23):5205-5208.

10. Baselga J, Cortes J, Kim SB, et al. Pertuzumab plus trastuzumab plus docetaxel for metastatic breast cancer. $N$ Engl J Med. 2012;366(2): 109-119.

11. Larkin J, Del Vecchio M, Ascierto PA, et al. Vemurafenib in patients with BRAF(V600) mutated metastatic melanoma: an open-label, multicentre, safety study. Lancet Oncol. 2014;15(4):436-444.

12. Blanke CD, Rankin C, Demetri GD, et al. Phase III randomized, intergroup trial assessing imatinib mesylate at two dose levels in patients with unresectable or metastatic gastrointestinal stromal tumors expressing the kit receptor tyrosine kinase: S0033. J Clin Oncol. 2008; 26(4):626-632.

13. Maemondo M, Inoue A, Kobayashi K, et al. Gefitinib or chemotherapy for non-small-cell lung cancer with mutated EGFR. N Engl J Med. 2010; 362(25):2380-2388.

14. Cancer Genome Atlas Research Network. Integrated genomic characterization of papillary thyroid carcinoma. Cell. 2014;159(3):676-690.

15. Ferrara N, Gerber HP, LeCouter J. The biology of VEGF and its receptors. Nat Med. 2003;9(6):669-676.

16. Yu XM, Lo CY, Lam AK, Leung P, Luk JM. Serum vascular endothelial growth factor $\mathrm{C}$ correlates with lymph node metastases and high-risk tumor profiles in papillary thyroid carcinoma. Ann Surg. 2008;247(3): 483-489.

17. Salajegheh A, Smith RA, Kasem K, et al. Single nucleotide polymorphisms and mRNA expression of VEGF-A in papillary thyroid carcinoma: potential markers for aggressive phenotypes. Eur J Surg Oncol. 2011;37(1):93-99.

18. Barone MV, Sepe L, Melillo RM, et al. RET/PTC1 oncogene signaling in PC $\mathrm{Cl} 3$ thyroid cells requires the small GTP-binding protein Rho. Oncogene. 2001;20(48):6973-6982.

19. St Bernard R, Zheng L, Liu W, Winer D, Asa SL, Ezzat S. Fibroblast growth factor receptors as molecular targets in thyroid carcinoma. Endocrinology. 2005;146(3):1145-1153.

20. Ricarte-Filho JC, Ryder M, Chitale DA, et al. Mutational profile of advanced primary and metastatic radioactive iodine-refractory thyroid cancers reveals distinct pathogenetic roles for BRAF, PIK3CA, and AKT1. Cancer Res. 2009;69(11):4885-4893.

21. Komminoth P. The RET proto-oncogene in medullary and papillary thyroid carcinoma. Molecular features, pathophysiology and clinical implications. Virchows Arch. 1997;431(1):1-9.

22. Chen KT, Lin JD, Liou MJ, Weng HF, Chang CA, Chan EC. An aberrant autocrine activation of the platelet-derived growth factor alpha-receptor in follicular and papillary thyroid carcinoma cell lines. Cancer Lett. 2006;231(2):192-205

23. Zhang J, Wang P, Dykstra M, et al. Platelet-derived growth factor receptor-alpha promotes lymphatic metastases in papillary thyroid cancer. J Pathol. 2012;228(2):241-250.

24. Rivera M, Ricarte-Filho J, Knauf J, et al. Molecular genotyping of papillary thyroid carcinoma follicular variant according to its histological subtypes (encapsulated vs infiltrative) reveals distinct BRAF and RAS mutation patterns. Mod Pathol. 2010;23(9):1191-1200.
25. Wilhelm SM, Carter C, Tang L, et al. BAY 43-9006 exhibits broad spectrum oral antitumor activity and targets the RAF/MEK/ERK pathway and receptor tyrosine kinases involved in tumor progression and angiogenesis. Cancer Res. 2004;64(19):7099-7109.

26. Carlomagno F, Anaganti S, Guida T, et al. BAY 43-9006 inhibition of oncogenic RET mutants. J Natl Cancer Inst. 2006;98(5):326-334.

27. Brose MS, Nutting CM, Jarzab B, et al. Sorafenib in radioactive iodine-refractory, locally advanced or metastatic differentiated thyroid cancer: a randomised, double-blind, phase 3 trial. Lancet. 2014; 384(9940):319-328.

28. Ferrari SM, Politti U, Spisni R, et al. Sorafenib in the treatment of thyroid cancer. Expert Rev Anticancer Ther. 2015;15(8):863-874.

29. Zhang L, Zhou Q, Ma L, Wu Z, Wang Y. Meta-analysis of dermatological toxicities associated with sorafenib. Clin Exp Dermatol. 2011; 36(4):344-350.

30. Ren Z, Zhu K, Kang H, et al. Randomized controlled trial of the prophylactic effect of urea-based cream on sorafenib-associated handfoot skin reactions in patients with advanced hepatocellular carcinoma. J Clin Oncol. 2015;33(8):894-900.

31. Santoni M, Conti A, De Giorgi U, et al. Risk of gastrointestinal events with sorafenib, sunitinib and pazopanib in patients with solid tumors: a systematic review and meta-analysis of clinical trials. Int J Cancer. 2014;135(4):763-773.

32. Matsui J, Funahashi Y, Uenaka T, Watanabe T, Tsuruoka A, Asada M. Multi-kinase inhibitor E7080 suppresses lymph node and lung metastases of human mammary breast tumor MDA-MB-231 via inhibition of vascular endothelial growth factor-receptor (VEGF-R) 2 and VEGF-R3 kinase. Clin Cancer Res. 2008;14(17):5459-5465.

33. Matsui J, Yamamoto Y, Funahashi Y, et al. E7080, a novel inhibitor that targets multiple kinases, has potent antitumor activities against stem cell factor producing human small cell lung cancer H146, based on angiogenesis inhibition. Int J Cancer. 2008;122(3):664-671.

34. Okamoto K, Kodama K, Takase K, et al. Antitumor activities of the targeted multi-tyrosine kinase inhibitor lenvatinib (E7080) against RET gene fusion-driven tumor models. Cancer Lett. 2013;340(1): 97-103.

35. Yamamoto Y, Matsui J, Matsushima T, et al. Lenvatinib, an angiogenesis inhibitor targeting VEGFR/FGFR, shows broad antitumor activity in human tumor xenograft models associated with microvessel density and pericyte coverage. Vasc Cell. 2014;6:18.

36. Tohyama O, Matsui J, Kodama K, et al. Antitumor activity of lenvatinib (e 7080): an angiogenesis inhibitor that targets multiple receptor tyrosine kinases in preclinical human thyroid cancer models. J Thyroid Res. 2014;2014:638747.

37. Glen H, Mason S, Patel H, Macleod K, Brunton VG. E7080, a multitargeted tyrosine kinase inhibitor suppresses tumor cell migration and invasion. BMC Cancer. 2011;11:309.

38. GivolD, Yayon A. Complexity ofFGF receptors: genetic basis for structural diversity and functional specificity. FASEB J. 1992;6(15)3362-3369.

39. Shingu K, Fujimori M, Ito K, et al. Expression of fibroblast growth factor-2 and fibroblast growth factor receptor-1 in thyroid diseases: difference between neoplasms and hyperplastic lesions. Endocr J. 1998; 45(1):35-43.

40. Onose H, Emoto N, Sugihara H, Shimizu K, Wakabayashi I. Overexpression of fibroblast growth factor receptor 3 in a human thyroid carcinoma cell line results in overgrowth of the confluent cultures. Eur J Endocrinol. 1999;140(2):169-173.

41. Koziczak M, Holbro T, Hynes NE. Blocking of FGFR signaling inhibits breast cancer cell proliferation through downregulation of D-type cyclins. Oncogene. 2004;23(20):3501-3508.

42. Kondo T, Zheng L, Liu W, Kurebayashi J, Asa SL, Ezzat S. Epigenetically controlled fibroblast growth factor receptor 2 signaling imposes on the RAS/BRAF/mitogen-activated protein kinase pathway to modulate thyroid cancer progression. Cancer Res. 2007;67(11): 5461-5470.

43. Yamada K, Yamamoto N, Yamada Y, et al. Phase I dose-escalation study and biomarker analysis of E7080 in patients with advanced solid tumors. Clin Cancer Res. 2011;17(8):2528-2537. 
44. Boss DS, Glen H, Beijnen JH, et al. A phase I study of E7080, a multitargeted tyrosine kinase inhibitor, in patients with advanced solid tumours. Br J Cancer. 2012;106(10):1598-1604.

45. Cabanillas ME, Schlumberger M, Jarzab B, et al. A phase 2 trial of lenvatinib (E7080) in advanced, progressive, radioiodine-refractory, differentiated thyroid cancer: a clinical outcomes and biomarker assessment. Cancer. 2015;121(16):2749-2756.

46. Schlumberger M, Jarzab B, Cabanillas ME, et al. A phase 2 trial of the multi-targeted tyrosine kinase inhibitor lenvatinib (E7080) in advanced medullary thyroid cancer (MTC). Clin Cancer Res. 2016:22(1): 44-53.

47. Schlumberger M, Tahara M, Wirth LJ, et al. Lenvatinib versus placebo in radioiodine-refractory thyroid cancer. $N$ Engl J Med. 2015; 372(7):621-630

48. Kate Newbold RE, Taylor MH, Krzyzanowska MK, et al. Efficacy and safety of lenvatinib for the treatment of patients with131Irefractory differentiated thyroid cancer with and without prior VEGFtargeted therapy. ASCO Annual Meeting 2015. J Cli Oncol. 2015; 33(Suppl):Abstract 6013.

49. Shumaker RC, Zhou M, Ren M, et al. Effect of lenvatinib (E7080) on the QTc interval: results from a thorough QT study in healthy volunteers. Cancer Chemother Pharmacol. 2014;73(6):1109-1117.

50. Guo MS, S, Wirth L, et al. Overall survival gain with lenvatinib vs. placebo in radioactive iodine refractory differentiated thyroid cancer (RR-DTC): an updated analysis. 18th ECCO - 40th ESMO European Cancer Congress, Proffered Paper Session, September, 2015, Vienna, Austria.

51. Shah RR, Morganroth J. Update on cardiovascular safety of tyrosine kinase inhibitors: with a special focus on QT interval, left ventricular dysfunction and overall risk/benefit. Drug Saf. 2015;38(8): 693-710

52. Lenvima [package insert]. Eisai Inc. under license from Eisai R\&D Management Co., Ltd.

53. Blevins DP, Dadu R, Hu M, et al. Aerodigestive fistula formation as a rare side effect of antiangiogenic tyrosine kinase inhibitor therapy for thyroid cancer. Thyroid. 2014;24(5):918-922.

54. Basille D, Andrejak M, Bentayeb H, et al. Bronchial fistula associated with sunitinib in a patient previously treated with radiation therapy. Ann Pharmacother. 2010;44(2):383-386.

55. Gore E, Currey A, Choong N. Tracheoesophageal fistula associated with bevacizumab 21 months after completion of radiation therapy. J Thorac Oncol. 2009;4(12):1590-1591.

56. Schutz FA, Je Y, Richards CJ, Choueiri TK. Meta-analysis of randomized controlled trials for the incidence and risk of treatment-related mortality in patients with cancer treated with vascular endothelial growth factor tyrosine kinase inhibitors. J Clin Oncol. 2012;30(8):871-877.

57. Kimura ET, Nikiforova MN, Zhu Z, Knauf JA, Nikiforov YE, Fagin JA. High prevalence of BRAF mutations in thyroid cancer: genetic evidence for constitutive activation of the RET/PTC-RAS-BRAF signaling pathway in papillary thyroid carcinoma. Cancer Res. 2003; 63(7):1454-1457.

58. Hou P, Liu D, Xing M. Functional characterization of the T17991801del and A1799-1816ins BRAF mutations in papillary thyroid cancer. Cell Cycle. 2007;6(3):377-379.

59. Xing M, Liu R, Liu X, et al. BRAF V600E and TERT promoter mutations cooperatively identify the most aggressive papillary thyroid cancer with highest recurrence. J Clin Oncol. 2014;32(25):2718-2726.

60. Liu Z, Hou P, Ji M, et al. Highly prevalent genetic alterations in receptor tyrosine kinases and phosphatidylinositol 3-kinase/akt and mitogenactivated protein kinase pathways in anaplastic and follicular thyroid cancers. J Clin Endocrinol Metab. 2008;93(8):3106-3116.

61. Abubaker J, Jehan Z, Bavi P, et al. Clinicopathological analysis of papillary thyroid cancer with PIK3CA alterations in a Middle Eastern population. J Clin Endocrinol Metab. 2008;93(2):611-618.

62. Schlumberger M, Massicotte MH, Nascimento CL, Chougnet C, Baudin E, Leboulleux S. Kinase inhibitors for advanced medullary thyroid carcinoma. Clinics (Sao Paulo). 2012;67(suppl 1): $125-129$.
63. Bunone G, Vigneri P, Mariani L, et al. Expression of angiogenesis stimulators and inhibitors in human thyroid tumors and correlation with clinical pathological features. Am J Pathol. 1999;155(6): 1967-1976.

64. Capp C, Wajner SM, Siqueira DR, Brasil BA, Meurer L, Maia AL. Increased expression of vascular endothelial growth factor and its receptors, VEGFR-1 and VEGFR-2, in medullary thyroid carcinoma. Thyroid. 2010;20(8):863-871.

65. Rodriguez-Antona C, Pallares J, Montero-Conde C, et al. Overexpression and activation of EGFR and VEGFR2 in medullary thyroid carcinomas is related to metastasis. Endocr Relat Cancer. 2010;17(1):7-16.

66. Makoto Tahara MS, Rossella E, Mouhammed AH, et al. Pharmacodynamic biomarkers of outcomes in the phase III study of lenvatinib in 131I-refractory differentiated thyroid cancer (SELECT). ASCO Annual Meeting 2015. J Clin Oncol. 2015;33(Suppl):Abstract 6014.

67. Marcia S, Brose MS, Makoto T, et al. Effect of age and lenvatinib treatment on overall survival for patients with 131I-refractory differentiated thyroid cancer in SELECT. ASCO Annual Meeting 2015. J Clin Oncol. 2015;33(Suppl):Abstract 6048.

68. Haugen BRM, Alexander EK, Bible KC, et al. 2015 American Thyroid Association Management Guidelines for Adult Patients with Thyroid Nodules and Differentiated Thyroid Cancer. Thyroid. 2015.

69. Guidelines N. NCCN Clinical Practice Guidelines in Oncology (NCCN Guidelines $^{\circledR}$ ) Version 2.2015. Available from: http://www.nccn.org/ professionals/physician_gls/pdf/thyroid.pdf. Accessed December 20, 2015.

70. Touat M, Ileana E, Postel-Vinay S, Andre F, Soria JC. Targeting FGFR signaling in cancer. Clin Cancer Res. 2015;21(12):2684-2694.

71. Schlumberger BJ, Elisei R1, Siena S, et al. Phase III randomized, doubleblinded, placebo-controlled trial of sorafenib in locally advanced or metastatic patients with radioactive iodine (RAI)-refractory differentiated thyroid cancer (DCT)-Exploratory analyses of patient-reported outcomes. 83rd American Thyroid Association Meeting. 2013.

72. Dadu R, Devine C, Hernandez M, et al. Role of salvage targeted therapy in differentiated thyroid cancer patients who failed first-line sorafenib. J Clin Endocrinol Metab. 2014;99(6):2086-2094.

73. ClinicalTrial.gov [database on the Internet]. Dabrafenib with or without trametinib in treating patients with recurrent thyroid cancer. Accessed October 3, 2015.

74. Ruco LP, Ranalli T, Marzullo A, et al. Expression of Met protein in thyroid tumours. J Pathol. 1996;180(3):266-270.

75. Koo BS, Kim JM, Seo ST, et al. Upregulation of HGF and c-MET is associated with subclinical central lymph node metastasis in papillary thyroid microcarcinoma. Ann Surg Oncol. 2014;21(7):2310-2317.

76. Scarpino S, Stoppacciaro A, Colarossi C, et al. Hepatocyte growth factor (HGF) stimulates tumour invasiveness in papillary carcinoma of the thyroid. J Pathol. 1999;189(4):570-575.

77. Scarpino S, D’Alena FC, Di Napoli A, Ballarini F, Prat M, Ruco LP. Papillary carcinoma of the thyroid: evidence for a role for hepatocyte growth factor (HGF) in promoting tumour angiogenesis. J Pathol. 2003; 199(2):243-250.

78. Mineo R, Costantino A, Frasca F, et al. Activation of the hepatocyte growth factor (HGF)-Met system in papillary thyroid cancer: biological effects of HGF in thyroid cancer cells depend on Met expression levels. Endocrinology. 2004;145(9):4355-4365.

79. Chattopadhyay C, El-Naggar AK, Williams MD, Clayman GL. Small molecule c-MET inhibitor PHA665752: effect on cell growth and motility in papillary thyroid carcinoma. Head Neck. 2008;30(8): 991-1000

80. Nakagawa T, Matsushima T, Kawano S, et al. Lenvatinib in combination with golvatinib overcomes hepatocyte growth factor pathway-induced resistance to vascular endothelial growth factor receptor inhibitor. Cancer Sci. 2014;105(6):723-730.

81. ClinicalTrial.gov [database on the Internet]. E7050 in combination with E7080 in subjects with advanced solid tumors (dose escalation) and in subjects with recurrent glioblastoma or unresectable stage III or stage IV melanoma after prior systemic therapy (expansion cohort and phase 2). Accessed October 3, 2015. 
82. Dienstmann R, Brana I, Rodon J, Tabernero J. Toxicity as a biomarker of efficacy of molecular targeted therapies: focus on EGFR and VEGF inhibiting anticancer drugs. Oncologist. 2011;16(12):1729-1740.

83. Ravaud A, Schmidinger M. Clinical biomarkers of response in advanced renal cell carcinoma. Ann Oncol. 2013;24(12):2935-2942.

84. Koyama N, Saito K, Nishioka Y, et al. Pharmacodynamic change in plasma angiogenic proteins: a dose-escalation phase 1 study of the multi-kinase inhibitor lenvatinib. BMC Cancer. 2014;14:530.

85. Sim SH, Keam B, Kim DW, et al. The gefitinib dose reduction on survival outcomes in epidermal growth factor receptor mutant non-small cell lung cancer. J Cancer Res Clin Oncol. 2014;140(12):2135-2142.

86. Yeo WL, Riely GJ, Yeap BY, et al. Erlotinib at a dose of $25 \mathrm{mg}$ daily for non-small cell lung cancers with EGFR mutations. J Thorac Oncol. 2010;5(7):1048-1053.

87. Faber E, Divoka M, Skoumalova I, et al. A lower dosage of imatinib is sufficient to maintain undetectable disease in patients with chronic myeloid leukemia with long-term low-grade toxicity of the treatment. Leuk Lymphoma. 2016;57(2):1-6.

88. ClinicalTrial.gov [database on the Internet]. An expanded access program with lenvatinib for the treatment of radioiodine-refractory differentiated thyroid cancer. Accessed October 3, 2015.

89. Burtness B, Anadkat M, Basti S, et al . NCCN Task Force Report: management of dermatologic and other toxicities associated with EGFR inhibition in patients with cancer. J Natl Compr Canc Netw. 2009; 7(suppl 1):S5-S21.

90. Brose MS, Frenette CT, Keefe SM, Stein SM. Management of sorafenibrelated adverse events: a clinician's perspective. Semin Oncol. 2014; 41(suppl 2):S1-S16.

91. Carhill AA, Cabanillas ME, Jimenez C, et al. The non-investigational use of tyrosine kinase inhibitors in thyroid cancer: establishing a standard for patient safety and monitoring. J Clin Endocrinol Metab. 2013;98(1):31-42.

92. Carr LL, Mankoff DA, Goulart BH, et al. Phase II study of daily sunitinib in FDG-PET-positive, iodine-refractory differentiated thyroid cancer and metastatic medullary carcinoma of the thyroid with functional imaging correlation. Clin Cancer Res. 2010;16(21):5260-5268.

93. Cohen EE, Rosen LS, Vokes EE, et al. Axitinib is an active treatment for all histologic subtypes of advanced thyroid cancer: results from a phase II study. J Clin Oncol. 2008;26(29):4708-4713.

94. Bible KC, Suman VJ, Molina JR, et al. Efficacy of pazopanib in progressive, radioiodine-refractory, metastatic differentiated thyroid cancers: results of a phase 2 consortium study. Lancet Oncol. 2010;11(10): 962-972.

95. Sherman SI, Wirth LJ, Droz JP, et al. Motesanib diphosphate in progressive differentiated thyroid cancer. $N$ Engl J Med. 2008;359(1):31-42.

96. Cabanillas ME, Brose MS, Holland J, Ferguson KC, Sherman SI. A phase I study of cabozantinib (XL184) in patients with differentiated thyroid cancer. Thyroid. 2014;24(10):1508-1514.

97. Leboulleux S, Bastholt L, Krause T, et al. Vandetanib in locally advanced or metastatic differentiated thyroid cancer: a randomised, double-blind, phase 2 trial. Lancet Oncol. 2012;13(9):897-905.
98. Brose MS CM, Cohen EEW, Wirth L, et al. An open-label, multicenter phase 2 study of the BRAF inhibitor vemurafenib in patients with metastatic or unresectable papillary thyroid cancer positive for the BRAF V600 mutation and resistant to radioactive iodine. Eur J Cancer. 2013;49:E17-7119.

99. Falchook GS, Millward M, Hong D, et al. BRAF inhibitor dabrafenib in patients with metastatic BRAF-mutant thyroid cancer. Thyroid. 2015;25(1):71-77.

100. Ho AL, Grewal RK, Leboeuf R, et al. Selumetinib-enhanced radioiodine uptake in advanced thyroid cancer. $N$ Engl J Med. 2013;368(7): 623-632.

101. Chow LQ, Eckhardt SG. Sunitinib: from rational design to clinical efficacy. J Clin Oncol. 2007;25(7):884-896.

102. Hu-Lowe D, Hallin M, Feeley R, et al. Characterization of potency and activity of the VEGF/PDGF receptor tyrosine kinase inhibitor AG013736. Am Assoc Cancer Ress. 2002;43:5357.

103. Kumar R, Knick VB, Rudolph SK, et al. Pharmacokineticpharmacodynamic correlation from mouse to human with pazopanib, a multikinase angiogenesis inhibitor with potent antitumor and antiangiogenic activity. Mol Cancer Ther. 2007;6(7):2012-2021.

104. Hurwitz HI, Dowlati A, Saini S, et al. Phase I trial of pazopanib in patients with advanced cancer. Clin Cancer Res. 2009;15(12):4220-4227.

105. Polverino A, Coxon A, Starnes C, et al. AMG 706, an oral, multikinase inhibitor that selectively targets vascular endothelial growth factor, platelet-derived growth factor, and kit receptors, potently inhibits angiogenesis and induces regression in tumor xenografts. Cancer Res. 2006;66(17):8715-8721.

106. Yakes FM, Chen J, Tan J, et al. Cabozantinib (XL184), a novel MET and VEGFR2 inhibitor, simultaneously suppresses metastasis, angiogenesis, and tumor growth. Mol Cancer Ther. 2011;10(12):2298-2308.

107. Fallahi P, Ferrari SM, Di Bari F, et al. Cabozantinib in thyroid cancer. Recent Pat Anticancer Drug Discov. 2015;10(3):259-269.

108. Wedge SR, Ogilvie DJ, Dukes M, et al. ZD6474 inhibits vascular endothelial growth factor signaling, angiogenesis, and tumor growth following oral administration. Cancer Res. 2002;62(16):4645-4655.

109. Carlomagno F, Vitagliano D, Guida T, et al. ZD6474, an orally available inhibitor of KDR tyrosine kinase activity, efficiently blocks oncogenic RET kinases. Cancer Res. 2002;62(24):7284-7290.

110. Salerno P, De Falco V, Tamburrino A, et al. Cytostatic activity of adenosine triphosphate-competitive kinase inhibitors in BRAF mutant thyroid carcinoma cells. J Clin Endocrinol Metab. 2010;95(1): $450-455$.

111. Banerji U, Camidge DR, Verheul HM, et al. The first-in-human study of the hydrogen sulfate (Hyd-sulfate) capsule of the MEK1/2 inhibitor AZD6244 (ARRY-142886): a phase I open-label multicenter trial in patients with advanced cancer. Clin Cancer Res. 2010;16(5): 1613-1623.

112. Bollag G, Hirth P, Tsai J, et al. Clinical efficacy of a RAF inhibitor needs broad target blockade in BRAF-mutant melanoma. Nature. 2010;467(7315):596-599.
Drug Design, Development and Therapy

\section{Publish your work in this journal}

Drug Design, Development and Therapy is an international, peerreviewed open-access journal that spans the spectrum of drug design and development through to clinical applications. Clinical outcomes, patient safety, and programs for the development and effective, safe, and sustained use of medicines are a feature of the journal, which

\section{Dovepress}

has also been accepted for indexing on PubMed Central. The manuscript management system is completely online and includes a very quick and fair peer-review system, which is all easy to use. Visit http://www.dovepress.com/testimonials.php to read real quotes from published authors. 